\title{
Exploring the origins of grey matter damage in multiple sclerosis
}

Calabrese, Massimiliano ; Magliozzi, Roberta ; Ciccarelli, Olga ; Geurts, Jeroen J G ; Reynolds, Richard ; Martin, Roland

\begin{abstract}
Multiple sclerosis is characterized at the gross pathological level by the presence of widespread focal demyelinating lesions of the myelin-rich white matter. However, it is becoming clear that grey matter is not spared, even during the earliest phases of the disease. Furthermore, grey matter damage may have an important role both in physical and cognitive disability. Grey matter pathology involves both inflammatory and neurodegenerative mechanisms, but the relationship between the two is unclear. Histological, immunological and neuroimaging studies have provided new insight in this rapidly expanding field, and form the basis of the most recent hypotheses on the pathogenesis of grey matter damage.
\end{abstract}

DOI: https://doi.org/10.1038/nrn3900

Posted at the Zurich Open Repository and Archive, University of Zurich

ZORA URL: https://doi.org/10.5167/uzh-111099

Journal Article

Accepted Version

Originally published at:

Calabrese, Massimiliano; Magliozzi, Roberta; Ciccarelli, Olga; Geurts, Jeroen J G; Reynolds, Richard; Martin, Roland (2015). Exploring the origins of grey matter damage in multiple sclerosis. Nature Reviews Neuroscience, 16(3):147-158.

DOI: https://doi.org/10.1038/nrn3900 


\title{
Exploring the origins of grey matter damage in multiple sclerosis
}

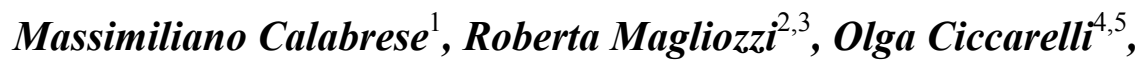 \\ Jeroen J.G. Geurts ${ }^{6}$, Richard Reynolds ${ }^{2}$ and Roland Martin ${ }^{7}$
}

Advanced Neuroimaging Lab of Neurology Section, Department of Neurological and Movement Sciences, University Hospital Verona, Piazzale LA Scuro 10, 37134, Verona, Italy

2 Division of Brain Sciences, Faculty of Medicine, Imperial College London, Hammersmith Hospital, London W12 0NN. United Kingdom.

3 Department of Cell Biology and Neuroscience, Istituto Superiore di Sanità, Viale Regina Elena 299 Rome, Italy

$4 \quad$ NIHR UCL/UCLH Biomedical Research Centre, London, United Kingdom

5 Queen Square MS Centre, University College London, Institute of Neurology, London, United Kingdom

6 Section of Clinical Neuroscience, Department of Anatomy \& Neurosciences, VU University Medical Center, van der Boechorststraat 7, 1081 BT Amsterdam

7 Neuroimmunology and MS Research Section, Department of Neurology, University Hospital Zurich, University Zurich, Frauenklinikstrasse 26, 8091 Zurich, Switzerland 


\begin{abstract}
Multiple sclerosis is characterised at the gross pathological level by the presence of widespread focal demyelinating lesions of the myelin-rich white matter. However, in recent years it has become clear that grey matter is not spared, even during the earliest phases of the disease. Furthermore, grey matter damage has been suggested to have an important role both in physical and cognitive disability. Grey matter pathology involves both inflammatory and neurodegenerative mechanisms, but the relationship between the two is unclear. This review will summarize and highlight important histological, immunological, and neuroimaging results from this rapidly expanding field and will address the most recent hypotheses on the pathogenesis of grey matter damage.
\end{abstract}

\title{
Introduction
}

Multiple Sclerosis is a chronic immune-mediated demyelinating disease of the central nervous system (CNS). Its aetiology has been suggested to involve both a complex genetic background with multiple susceptibility genes and several environmental factors ${ }^{1-4}$. Multiple sclerosis is characterized pathologically by inflammation, demyelination with partial restoration of myelin, axonal and neuronal damage and glial scarring ${ }^{5,6}$. The pathogenetic mechanisms leading to these abnormalities are largely unclear.

As disseminated focal demyelinating lesions in the white matter are the classical hallmark of multiple sclerosis, the disease has long been considered a 'typical white matter disease'. Nevertheless, early histological reports noted that grey matter is also affected and that grey matter damage and cell loss may underlie some of the permanent neurological dysfunction. In 1916, in a comprehensive overview of the histology of disseminated sclerosis, James W. Dawson wrote: “(...) when an area [of demyelination] is confined to the cortex, the changes are, as a rule, not nearly so marked (...).", , referring to the striking lack of glial proliferation and cellular paucity (an indicator that inflammation was absent) in grey matter lesions. Dawson also wondered: "Is then, the process that attacks the cortex different in its nature and origin from that which affects the rest of the central nervous system?" This tantalizing question remains highly relevant today.

There was little post-mortem tissue research investigating grey matter pathology in multiple sclerosis until 1962, when the interest in grey matter lesions in multiple sclerosis was rekindled by a seminal histopathology study ${ }^{8}$ that reported that $26 \%$ of multiple sclerosis lesions were located in or around the cortical and subcortical grey matter. As would later be apparent, this was a gross underestimation of actual grey matter damage. It was another four decades before advances in 
imaging techniques ${ }^{9,10}$, supported by immunohistochemical observations and animal models, renewed the interest in grey matter pathology in multiple sclerosis and began to reveal its high prevalence and to uncover the detailed cellular pathology of grey matter demyelination ${ }^{11-13}$.

However, despite the extensive pathological characterisation and the imaging evidence of grey matter lesions (Figure 1), the causes of grey matter damage and its relationship to white matter lesions remain unclear. This review aims to provide an overview of existing theories about grey matter damage in multiple sclerosis, answering, from a histological, immunological, and neuroimaging point of view, some of the most pertinent questions about its pathogenesis.

\section{Are white and grey matter damage linked?}

Since the first demonstration that the cortex is not spared by multiple sclerosis, the question of whether cortical tissue damage is secondary to white matter pathology - that is, whether it occurs via retrograde degeneration - or is a primary pathological process has remained unresolved.

Grey matter lesions are usually characterised by a relative lack of parenchymal lymphocyte infiltration, deposition of antibody and complement proteins, and blood brain barrier disruption when compared to white matter lesions ${ }^{12,14,15}$. In post mortem samples taken from individuals in the advanced stages of multiple sclerosis, high numbers of immune cells were only detected in Type I grey matter lesions (Figure 1A): all other grey matter lesions were categorized as relatively "noninflammatory"11-13. Moreover, according to post-mortem studies, neuronal loss occurs within both grey matter demyelinated lesions and normal appearing grey matter ${ }^{12,16,17}$, suggesting that neuronal loss might occur independently from grey matter demyelination.

These findings imply that grey matter might be damaged either from local inflammation that has occurred earlier within the grey matter or as a consequence of white matter lesions that are themselves caused by inflammation.

\section{Evidence that links grey and white matter damage}

In line with the idea that white matter damage may drive grey matter damage, several MRI studies have reported a significant correlation between white matter damage and grey matter atrophy in conditions other than multiple sclerosis ${ }^{18}$. For example, advanced post-processing analyses showed a reduction of cortical grey matter volume and thinning of the primary motor cortex in patients with spinal cord injury, suggesting that distant axonal transection can lead to significant cortical atrophy via retrograde degeneration ${ }^{18}$. 
It has been suggested that the characteristic pattern of white matter pathology in multiple sclerosis could lead to selective retrograde injury to frontal, temporal and motor cortical areas, which would explain some, but not all, of the post mortem patterns of grey matter damage that have been observed ${ }^{19}$. Indeed, the cortico-spinal tract, which contains fibre connections to the precentral gyrus, and the frontal periventricular white matter, which consists of efferent and afferent fibres to frontal and superior temporal lobes, are common sites of white matter lesions in multiple sclerosis $^{20}$. Moreover, axonal damage in active white matter lesions (i.e. gadolinium-enhanced on contrast MRI) could lead indirectly to anterograde and retrograde degeneration (Figure 2) of axons running within the thalamus and basal ganglia. Axon loss might also contribute to demyelination and/or Wallerian degeneration by reducing local metabolic activity ${ }^{21,22}$. A correlation between white matter lesion load and thalamic atrophy was found in patients with clinically isolated syndrome $^{23}$ and early relapsing remitting multiple sclerosis (RRMS) ${ }^{24}$. More generally, the assumption that white matter lesions and grey matter atrophy are linked is supported by several cross-sectional MRI studies showing significant correlations between the total grey matter volume and the total volume of white matter T1- and T2-weighted lesions ${ }^{25-28}$. Even when a more sensitive regional analysis was performed, a correlation between regional cortical grey matter loss and white matter lesion volume in the corresponding or adjacent lobes in progressive multiple sclerosis patients was observed ${ }^{29}$, although the location and extent of grey matter lesions were not studied. Finally, several longitudinal MRI studies ${ }^{30-32}$ point to an association between increasing white matter lesion volumes and grey matter volume loss in the frontal and parietal cortex of patients with RRMS. In addition, white matter lesion volume correlated with ventricular enlargement ${ }^{32}$, focal white matter damage in the optic radiations (see figure $3 \mathrm{~A}, 3 \mathrm{~B}, 3 \mathrm{C}$ ) and upstream grey matter atrophy of the lateral geniculate nucleus and visual cortex in patients with RRMS ${ }^{33}$.

\section{Evidence for independent grey matter damage}

An alternative but equally important theory suggests that white matter and grey matter demyelination are two, at least partly, independent phenomena and that neuronal loss is not caused by white matter abnormalities per se. Proponents of this theory suggest that retrograde changes as a result of focal white matter lesions do not satisfactorily explain the extent of the cellular pathology and diffuse nature of grey matter changes (Figure 3D, 3E, 3F compared to 3G, 3H, 3I).

Extensive pathological and imaging evidence supports this argument. For example, several recent studies of grey and white matter demyelination in different regions of the CNS found that the extent of cortical demyelination is greater than that found in white matter ${ }^{34}$ and that, although cortical demyelination sometimes occurred together with demyelination in the adjacent white matter (leukocortical lesions), in most instances, the cortex was affected independently from 
white matter lesions ${ }^{35,36}$. Another study observed a gradient of neuronal loss in the precentral gyrus of MS cases that exhibited extensive subpial demyelination with the greatest loss in the outer cortical layers $^{17}$. There was no relationship between this gradient and the white matter lesion volume or location, which argues strongly against an influence of white matter lesions on grey matter subpial pathology. On the contrary, it is likely that extensive primary neuronal loss in the cortical grey matter would lead to anterograde loss of axons in downstream white matter pathways, including the spinal cord.

It has also been shown that extensive and complete subpial demyelination of individual gyri and sulci accompanied by the relative preservation of axons characterises many cases of secondary progressive multiple sclerosis (SPMS) ${ }^{12,37}$. This pattern of damage cannot be explained by axonal damage in white matter lesions, which would lead to more diffuse but incomplete decrease in myelin density due to the loss of individual myelin-axon units. Extensive subpial demyelination was also observed in single biopsies of very early $\mathrm{RRMS}^{38}$. In vivo imaging studies confirmed the presence of grey matter lesions during the earliest phases of the disease ${ }^{39}$ in patients with very low white matter lesion volume, and sometimes even in patients with radiologically isolated syndrome (before any clinical symptoms are present ${ }^{40}$ ), or preceding the occurrence of white matter lesions altogether $^{41}$. However, it should be pointed out that even when using the most advanced technologies, most grey matter lesions (especially type 3 subpial lesions (figure 1C) escape identification by MRI, making it difficult to accurately correlate grey matter and white matter damage by this method ${ }^{42}$.

Other radiological data suggested that diffuse grey matter atrophy is only partially related to the white matter damage visible by MRI. When brain atrophy was studied in early RRMS patients, for example, both grey and white matter atrophy were observed, but the correlation between the two was only moderate ${ }^{43}$. A 2-year follow-up of these patients revealed that fluctuations in inflammatory white matter lesions were related to volume changes in white matter rather than to grey matter $\operatorname{loss}^{44}$. Voxel-based morphometry and tract-based spatial statistical analyses were applied to assess regional grey matter and white matter damage in 36 RRMS and 25 sex- and agematched controls. As expected, this revealed significant differences between patients and controls. However, although several measures of white matter damage correlated with each other, none correlated with grey matter volume ${ }^{45}$. More recent imaging studies have found that the relationship between grey matter atrophy and white matter abnormalities was weaker in primary and secondary progressive disease than in RRMS suggesting that neurodegenerative processes in patients with progressive disease are even less related to white matter changes ${ }^{46}$. These findings could indicate that grey matter damage is either independent of white matter lesions or that white matter is affected in a way that is not detected by currently applied imaging techniques. 
Finally, grey matter lesion probability maps and regional analysis of grey matter atrophy revealed a specific topographical distribution of focal and diffuse damage ${ }^{47}$. Most grey matter lesions were found in the fronto-temporal lobes, with a particular prevalence in the motor regions. High probability of focal grey matter demyelination was observed in the hippocampus, deep grey matter and insula ${ }^{48,49}$, whereas a significant increase in the distribution of diffuse subpial demyelination was observed by means of $7 \mathrm{~T}$ magnetic resonance in the anterior cingulate cortex ${ }^{50}$. Grey matter demyelination has also been observed in the limbic system and in specific cortical areas such as the mesial temporal lobe ${ }^{51}$. Nevertheless, the spatiotemporal topographical distribution of white matter lesions did not reveal any direct anatomical overlap between areas of significant grey matter volume reduction and significant focal white matter lesion volume increases $^{52}$.

Does grey matter damage occur as result of or independently of white matter damage? As described above, evidence exists for both. There is little doubt that axonal damage can lead to retrograde loss of neurons. However, it does not explain the spectrum and extent of grey matter damage in multiple sclerosis, and several studies clearly suggest that the mechanisms of damage within white matter and grey matter may be, at least partly, independent. Furthermore, primary damage to cortical neurons could give rise to anterograde axonal loss, particularly in the spinal cord.

\section{Inflammatory grey matter damage}

Although there is overall little information on the immune mechanisms that are active in the cortical layer in multiple sclerosis patients, several causes of inflammatory demyelination within the grey matter can be envisioned (Figure 4). These include the possible expression or relative overabundance of a target autoantigen for adaptive ( $\mathrm{T}$ cell- and antibody-mediated) immune mechanisms ${ }^{53}$ or the involvement of a $\mathrm{T}$ cell with specificity for both a myelin- and neuronal antigen $^{54}$. An alternative hypothesis suggests that the presence of inflammatory infiltrates in the meningeal space and/or in the adjacent perivascular spaces might lead to the release of cytotoxic inflammatory mediators into the grey matter ${ }_{-}^{17,38,55}$. This could be caused by an infectious organism located in the adjacent meninges or by a chronic compartmentalised inflammatory response to selfantigen(s). Several epidemiological studies have suggested that Epstein-Barr virus (EBV) is one of the strongest candidates for this infectious agent ${ }^{56-58}$ and EBV proteins and RNA have been detected in B-cells in the meninges and perivascular spaces of MS cases with extensive meningeal infiltrates and cortical demyelination ${ }^{59-61}$. It has been proposed that failure to control latent EBV infection in an immune privileged site, such as the subarachnoid space, could lead to recurrent 
intrathecal reactivation of EBV and tissue damage in the nearby grey matter ${ }^{62,63}$. However, a number of other studies have been unable to detect EBV in the brain or lesions of MS patients ${ }^{64,65}$ and this remains a highly debated controversy ${ }^{66}$. The different results found in these studies are likely to be due to the different types of tissues and MS cases used, the variable preservation of meningeal tissues in the samples, and differences in the sensitivity of the techniques used to detect EBV infection ${ }^{67}$. Other viruses, such as Torque Teno virus, have also been hypothesised to play a role in the pathogenesis of $\mathrm{MS}$, on the basis that they can infect the $\mathrm{CNS}^{68}$, are a target for cerebrospinal fluid (CSF)-infiltrating $\mathrm{T}$ cells isolated from multiple sclerosis patients ${ }^{69}$ and interact with $\mathrm{EBV}^{68,69}$. Furthermore ${ }_{2}$ myco-like viruses that are closely related to Torque Teno virus have recently been isolated from cow milk and the brains of MS patients providing an interesting link to epidemiological findings on the consumption of cow milk and the geographical prevalence rates of $\mathrm{MS}^{70}$. Alternatively, cortical demyelination and neuronal loss could involve an infectious agent with primary tropism for oligodendrocytes and/or cortical neurons ${ }^{71}$, although no evidence exists yet for this possibility in multiple sclerosis.

Other mechanisms that might target adaptive and/or innate immune responses to the grey matter include alterations specifically linked to the function and/or degeneration of neurons, astrocytes or oligodendrocytes, such as metabolic changes ${ }^{72}$, excitatory neurotransmitter release ${ }^{73}$, expression of post-translationally modified proteins and/or peptides ${ }^{74}$, changes in electrical activity and/or ion currents and cytokine and/or cytokine receptor expression ${ }^{75}$.

Although none of these possible reasons has yet been proven to be a cause of the topographical distribution of grey matter damage in multiple sclerosis, there are data in support of these ideas, and we therefore believe that they can at least serve as a working hypothesis and basis for further investigation.

\section{Role of parenchymal and perivascular lymphocytes}

A recent study demonstrated that the extent of lymphocyte infiltration in grey matter lesions depends on the disease stage: foamy macrophages, thought to be involved in ongoing demyelination, were found in grey matter active lesions of $66 \%$ of early RRMS patients at biopsy ${ }^{38}$ but were rarely found in grey matter lesions from SPMS patients ${ }^{37}$. The presence of perivascular CD8+ $\mathrm{T}$ cells was observed in $77 \%$ of intracortical lesions at biopsy with varying frequency depending on the location examined (with numbers decreasing from leucocortical regions to intracortical lesions and being lowest in subpial lesions ${ }^{12,38}$ ). Perivascular T- and B cell infiltrates have also been observed in both intracortical and sub-pial lesions in progressive multiple sclerosis $\operatorname{cases}^{55}$, particularly in subjects in whom progressive disease with signs of activate inflammation 
was present at death. The reasons for the heterogeneous distribution of CD8+ T cells in the cortex, their relatively higher frequency in leukocortical- and intracortical plaques in comparison to subpial ones and their localization in perivascular cuffs ${ }^{38}$ are at present not clear, and further studies are clearly needed.

A wealth of immunological data suggests that CD4+ $\mathrm{T}$ cells are inducers and drivers of multiple sclerosis ${ }^{2}$. Key findings that support this notion are among others the strong genetic influence of the HLA-DR15 haplotype ${ }^{76,77}$, the increased frequency of myelin-specific CD4+ T cells with a proinflammatory phenotype in $\mathrm{MS}^{78,79}$, and their higher antigen avidity ${ }^{80}$. However, although CD4+ T cells are present in the inflamed meninges above sub-pial grey matter lesions ${ }^{17}$, little information is available concerning their presence within cortical grey matter lesions. This is surprising if one considers that HLA-class II molecules (including HLA-DR alleles) serve as recognition structures for CD4+ T cells and the HLA-DR15 haplotype is by far the most important genetic risk factor in multiple sclerosis ${ }^{76,77}$. In fact, a recent study suggested that HLA-DRB $1 * 15$ status is associated with the extent of inflammation and demyelination in motor cortex at autopsy ${ }^{81}$, indirectly suggesting that CD4+ T cells (or an as yet unknown role of HLA-class II molecules) are involved in tissue inflammation in the grey matter.

Despite the evidence for a role for CD4 $+\mathrm{T}$ cells in multiple sclerosis, $\mathrm{CD} 8+\mathrm{T}$ cells are found at higher frequency than CD4+ T cells in the grey matter and brains of multiple sclerosis patients at autopsy ${ }^{38,82}$, and are more frequently clonally expanded, ${ }^{83}$ i.e. these cells have proliferated locally in the brain. However, the local proliferation within the grey matter lesions themselves has not been examined yet, and CD4+ intracerebral T cells have generally been studied less well. CD8+ T cells are also thought to be more likely to directly damage neurons and other CNS cells than CD4+ T cells because HLA-class II molecules are expressed at very low levels and on a limited number of cells in the CNS, whereas HLA-class I molecules, which present antigen to CD8+ T cells, show broader expression in multiple sclerosis brain $^{75}$. However, although HLA-class II molecules are not expressed by neurons, they are expressed by activated microglia and astrocytes. Proteins and peptides that are released from damaged neurons can be presented by these cells to CD4+ T cells ${ }^{84}$, although this process has not been examined yet in the grey matter of multiple sclerosis patients. Furthermore, HLA-class I molecule expression has been described in electrically silent neurons (those that are functionally compromised or damaged) and data from experimental systems suggests that the exposure of neurons to interferon-g (IFN-g) renders them immunological targets for CD8+ $\mathrm{T}_{\text {cells }}{ }^{75}$. In summary, the existing evidence suggests an involvement of both CD4+ and CD8+ T cells in cortical damage in multiple sclerosis albeit with different contributions, i.e. initiation and perpetuation by CD4+ $\mathrm{T}$ cells and effector mechanisms such as direct neuronal/axonal damage more likely by CD8+ T cells. 
Several mechanisms through which CD8+ and CD4+ $\mathrm{T}$ cells could damage neurons and oligodendrocytes have been proposed. These include antigen-specific and antigen-independent mechanisms as well as cell contact-dependent and -independent processes ${ }^{85}$. However, all of the major cytotoxic mechanisms that have been proposed - including the release of perforin ${ }^{86}$ or granzymes $^{87}$, involvement of Fas/Fas-ligand ${ }^{88}$, cell lysis by TNF receptor-related apoptosisinducing ligand (TRAIL) ${ }^{89}$ and the induction by IFN-g of neuron-specific, calcium-permeable complexes between IFN-g-receptor and the glutamate receptor GluR $1^{90}$ - have been described mainly in experimental models. It is important to note that the ability to kill target cells by perforinor Fas/Fas-ligand-mediated lysis, which is typically attributed only to CD8+ T cells, has also been shown for human CD4+ $\mathrm{T}$ cells ${ }^{91}$. Furthermore, an antigen-independent lysis mechanism that involves CD56 (or neural cell adhesion molecule, NCAM), which is expressed on natural killer cells and a subset of CD4+ T cells ${ }^{92}$, has been implicated in the lysis of oligodendrocytes ${ }^{93}$.

\section{Role of meningeal inflammatory infiltrates}

In the last decade several neuropathology studies have shed light on the association between meningeal inflammation and pathology of the adjacent cerebral cortex, both in multiple sclerosis and in experimental autoimmune encephalomyelitis (EAE) models ${ }^{17,38,94-98}$. Aberrant tertiary lymphoid-like structures containing large aggregates of $\mathrm{CD} 20^{+}$B-cells ${ }^{17,37,94}$, which are hallmarks of several other chronic inflammatory diseases, were found in the inflamed meninges of a substantial proportion of SPMS cases that were examined post-mortem. Similar accumulations of B-cells have been reported in 40\% of cortical biopsies from early RRMS cases, where they are associated with underlying subpial demyelination ${ }^{38}$. The SPMS cases exhibiting lymphoid-like immune cell aggregates were characterized by a high degree of inflammatory activity ${ }^{17,37,94,99}$. The study was carried out on a large number (123) of post-mortem MS cases with a wide range of ages at onset, progression and death and total disease duration in order to avoid selection bias ${ }^{37}$. However, an independent smaller study failed to find such lymphoid structures ${ }^{100}$. As has previously been discussed, this disparity may be a result of differences in the technical approaches used, such as different procedures of tissue processing, cutting and staining, and cohort choice. ${ }^{65,101}$. The presence of organised meningeal infiltrates occurred in the main in MS cases characterised by an earlier age at death and evidence of ongoing inflammatory activity and such cases are not always present in autopsy collections. In addition to the organised lymphoid-like structures that have been reported, extensive diffuse meningeal infiltrates have been detected at autopsy, ${ }^{100,102,103}$ which may explain the widespread nature of subpial demyelination. Subpial demyelination and cortical atrophy are more pronounced within deep invaginations of the cortex ${ }^{16,17,35}$, suggesting that regional differences in CSF flow and/or stasis may result in a shielded niche (or microenvironment) for the 
persistence of lymphoid-like structures within cerebral sulci. It can be speculated that this may sustain a local immune response - particularly enriched in $\mathrm{CD} 20^{+} \mathrm{B}$ lymphocytes and plasmablasts, but also comprising $\mathrm{CD} 4+$ and $\mathrm{CD} 8+\mathrm{T}$ cells and macrophages - that chronically generates inflammatory, cytotoxic and possibly myelinotoxic mediators that, by circulating within the CSF, may diffuse freely throughout the subarachnoid space. These mediators might cross the pial membrane towards the adjacent grey matter and specifically mediate diffuse and focal subpial grey matter injury in multiple sclerosis. The release and circulation of specific, but still as yet unknown factors, in the CSF bathing the cerebral cortex could explain the fact that subpial cortical demyelination is one of the most specific features of MS pathology ${ }^{104}$. Among these factors tumour necrosis factor (TNF) and IFN- $\gamma$, whose expression was found significantly increased both in the meninges and CSF samples from post-mortem SPMS cases with higher levels of meningeal inflammation and cortical damage ${ }^{99}$, may play a fundamental role in mediating subpial pathology directly or indirectly, by regulating microglia activity. This hypothesis is corroborated by the finding that the subset of SPMS patients with higher levels of inflammation and frequency of immune cell infiltrates in the meninges had a higher subpial grey matter lesion volume, a higher degree of neuronal, astrocyte and oligodendrocyte loss and increased microglial activation ${ }^{17,37}$, the severity of which followed a gradient from the external towards the innermost cortical layers.

Interestingly, the subpopulation of SPMS cases with higher levels of meningeal inflammation and grey matter damage were characterised clinically by a more rapidly progressive disease course $^{17,37,94,105}$, although it should be noted that this population of patients clearly represent the more aggressive end of the spectrum of heterogeneous presentations. The link between cortical pathology and a rapidly progressive disease course has also been described in a large proportion of multiple sclerosis cases using non-conventional imaging techniques ${ }^{39,105}$. These results support the idea that increased grey matter pathology, alone or in addition to white matter pathology, may be associated with a more rapid and aggressive disease course from early phases of the disease and that meningeal inflammation may in part be responsible for this increased pathology.

The specific link between meningeal inflammation and severity of grey matter pathology and rapidly progressive disease course is further supported by studies of the spinal cord of post-mortem cases with secondary progressive multiple sclerosis ${ }^{106}$, where the levels of both meningeal $\mathrm{T}$ cells and activated parenchymal microglia were associated with increased diffuse axonal loss. Furthermore the recent examination of a large number of brain biopsies from patients with recently diagnosed multiple sclerosis ${ }^{38}$ has indeed revealed that cortical lesions occur early in the disease and are highly inflammatory and associated with both focal perivascular and diffuse meningeal inflammation. The reported association between meningeal inflammation and immune infiltrates in chronic subpial lesions, either in brain biopsies from patients with early multiple sclerosis ${ }^{38}$ or in 
post-mortem SPMS cases with more aggressive grey matter pathology ${ }^{94}$, indicate that both meningeal and intraparenchymal grey matter inflammation may play a role in disease pathogenesis, either by causing cortical damage at the beginning of the disease and/or by exacerbating grey matter pathology as disease progresses

\section{Role of microglia}

Microglia are innate immune cells that are resident in the CNS and are considered to be the dominant effector cell population in cortical grey matter injury ${ }^{12}$. Elongated microglia that are oriented perpendicularly to the pial surface and are closely apposed to apical dendrites and axons, together with activated stellate microglia that extend processes to neuronal perikarya, dendrites and axons, are observed in active and chronic grey matter lesions ${ }^{107}$. The number of activated microglia and the degree of activation have been shown to correlate with the density of transected neurites in grey matter lesions ${ }^{12}$, suggesting that dendrites and axons are vulnerable to microglial activation ${ }^{107}$. Microglial activation could also be a consequence of neuronal and axonal damage and thus a sign of prior injury ${ }^{108}$. However, in post-mortem brain tissue, it has been shown that the number of microglia in cortical lesions also correlates with the degree of meningeal immune cell infiltration ${ }^{37}$. Therefore, it is unclear whether microglia have a neuroprotective and/or detrimental role within the cortex in multiple sclerosis or what the sequence of events is.

In the mature brain microglia typically exist in a resting state and continually survey the microenvironment with their motile processes and protrusions ${ }^{109}$. Blood brain barrier disruption, brain injury or immunological stimuli provoke immediate and focal activation of microglia ${ }^{110}$. Depending on the circumstances of activation, microglia may differentiate into a type 1 phenotype, which can be proinflammatory and detrimental, or a type 2 phenotype, which is immunomodulatory, supports survival and provides an anti-oxidant defence ${ }^{111}$. However, the phenotype of activated microglia in cortical multiple sclerosis lesions has yet to be determined. In order for microglia to remain in a chronic state of activation in the multiple sclerosis cortex, there would need to be a chronic stimulus. It can be hypothesised that the presence of a chronically inflammatory milieu in the subarachnoid ${ }^{99}$ and perivascular spaces ${ }^{61}$ might represent such a stimulus. Other stimuli that promote microglial over-activation and dysregulation include environmental toxins and neuronal death or damage. However, the conditions that determine whether microglial activation is detrimental or beneficial to neuronal survival are currently poorly understood in multiple sclerosis.

Nevertheless, it is becoming more widely accepted that, whereas microglial activation is necessary and crucial for host defence and neuronal survival, their over-activation may be deleterious to neurons and oligodendrocytes ${ }^{12}$. In postmortem homogenates of demyelinated and 
non-demyelinated cerebral cortical regions from multiple sclerosis cases, grey matter demyelination was found to be associated with increased activity of myeloperoxidase, which is expressed by a CD68-positive subset of activated microglia found in active GM demyelination toward the edge of the lesions but not by microglia in adjacent non-demyelinated cortex ${ }^{113}$. Moreover, the presence of activated microglia in grey matter lesions in multiple sclerosis patients has been correlated with focal loss of the glutamate transporters, EAAT1 and EAAT2, and synaptophysin immunostaining ${ }^{114}$, suggesting that activated microglia may also perturb astrocyte function and glutamate metabolism. Alterations in the mechanisms of glutamate reuptake found in grey matter lesions in the presence of activated microglia could be associated with signs of neuronal and synaptic damage suggestive of excitotoxicity ${ }^{114}$ (see below). Furthermore, an increase in microglial activity together with neuronal and axonal loss has been found in subpial cortex of SPMS patients with increased diffuse and organised meningeal inflammation ${ }^{37,94}$. Increases in the numbers of activated CD68+ microglia have also been observed in active and/or chronic cortical lesions of primary progressive multiple sclerosis patients at autopsy ${ }^{103}$, and rims of activated microglia are found at the border of grey matter lesions in a large fraction of multiple sclerosis patients with extensive subpial demyelination ${ }^{115}$. These patients were younger at time of death than patients without grey matter lesions or patients without rims of activated microglia in grey matter lesions ${ }^{100}$.

\section{Non-inflammatory neurodegeneration?}

A recent theory points to inconsistencies in the inflammatory model described above and suggests instead a degenerative model as the primary cause of the disease.

This model proposes that a primary 'cytodegeneration', initially focused on oligodendrocytes and/or neurons, begins years before any clinical symptoms. The autoimmune inflammatory reaction that is later observed would depend on the host's predilection to react to the antigens released as a consequence of the (neuro)degeneration. This hypothesis arose as a result of inconsistencies in the literature, including observations of early myelin protein degradation before the prototypical adaptive immune response and the inability to stop disease progression using potent anti-inflammatory drugs ${ }^{116-118}$.

However, although this hypothesis seems plausible, other interpretations are more likely: for example, adaptive immune processes that initiate white matter and grey matter damage may laterwhen demyelination and indirect injury to axons as well as direct axonal transections have reached a critical level - lead to on-going neurodegeneration that progresses independently of new inflammation. Alternatively, the efficacy of current immunomodulatory therapies may decrease as the immune response that drives multiple sclerosis pathology becomes increasingly compartmentalised within the CNS or CSF. According to this hypothesis, on-going inflammation 
would require less infiltration of cells from the peripheral immune system and therefore might not respond to systemically delivered therapies that primarily act as immunomodulatory in the peripheral immune system. It is likely, as has been suggested previously ${ }^{116}$, that multiple sclerosis involves both 'inside-out' and 'outside-in' disease mechanisms, jointly contributing to its variable course. However, large genome-wide association studies support a primary role of immune system alterations, since the vast majority of the more than 100 common genetic variants $^{119}$, that have identified so far are immune-related.

\section{Conclusions and Perspectives}

The evidence reviewed above suggests that damage to the grey matter in multiple sclerosis proceeds in a manner that is in part independent of white matter damage, although there is clearly some degree of relationship between them (Box 1). This relative independence is illustrated by the fact that some cases of multiple sclerosis exhibit predominantly grey matter pathology with little white matter involvement and vice versa. Neuronal degeneration inevitably follows axonal transection in white matter lesions and will lead to a variable and dispersed loss of neurons in grey matter areas related to the anatomical location of the white matter lesions. Neuronal loss in the grey matter, as a result of inflammatory processes or more intrinsic mechanisms of degeneration (i.e. a local energy deficit due to mitochondrial dysfunction, Box 2), will also lead to axon loss and degeneration of downstream neurons. Whether grey matter damage may, at some point in the disease, proceed due to a primary degenerative process independent of inflammation and independent of white matter damage remains to be resolved: at present there is no evidence of a primary trigger similar to that seen in other neurodegenerative conditions. Evidence exists for a role of inflammatory processes, both innate in the parenchyma and adaptive in the meninges and perivascular spaces, alongside a role for degenerative processes involving mitochondrial dysfunction and energy deficits. However, these different processes have yet to be fully linked. Studies of the very early stages of the disease are required to clarify whether inflammation and/or immune mechanisms or neurodegeneration is the primary cause of the disease, but it is already clear that at some point both mechanisms are simultaneously active in grey matter and white matter in multiple sclerosis. To complicate matters further, the relationship between white and grey matter damage may differ across brain regions, disease phases and multiple sclerosis phenotypes. Additional longitudinal studies that focus on the very early stages of the disease, when either the white matter or the grey matter are almost intact, are required to clarify the relationship between white and grey matter damage. Since such studies are unlikely to be possible using post-mortem or biopsy tissue, the most promising approaches will be combinations of ever more sophisticated 
imaging methods such as MRI, spectroscopy and PET imaging with immunological, proteomics and metabolomics methods using CSF.

In the light of accumulating evidence that multiple sclerosis patients with similar white matter lesion volumes undergo a more severe clinical course in presence of higher and more diffuse grey matter lesion volumes and atrophy already from the early disease phases, it may be suggested that cortical pathology has a key role in determining the rate of disability progression in multiple sclerosis. As with other progressive neurodegenerative conditions, it is likely that the accumulation of disability is directly related to increasing loss of vital neuronal populations and the rate at which they are lost and their locations determine the relative rate of disease progression. However, unlike other neurodegenerative conditions, the loss of neurons and axons is much more diffuse, which is reflected in the heterogeneous decline in diverse motor, sensory and cognitive function. Knowledge of the mechanisms underlying grey matter damage and identification of measures of disease progression associated with cortical pathology could help identify prognostic biomarkers and individualized therapies with increased efficacy for patients with a predisposition to develop more severe cortical pathology and associated clinical deterioration. Whether this predisposition is linked to genetic, environmental or other factors and is common to white matter immunopathogenesis is still unknown and highlights the need for more detailed study. Despite the unknowns, from a therapeutic point of view, a combination of immunomodulatory and neuroprotective strategies appears most promising to limit grey matter degeneration and development of long-term disability.

\section{BOX 1: Possible mechanisms underlying grey matter damage}

\section{Adaptive immunity and /or autoimmune mechanisms}

\section{a) Auto-antigens}

The over-expression of specific auto-antigens, epitope spreading to antigens that are more abundantly expressed in the grey matter, post-translational modifications of molecules in the grey matter, or molecular mimicry between myelin- and neuronal antigens has been hypothesised to activate $\mathrm{T}$ cells (preferentially CD8+ cytotoxic $\mathrm{T}$ cells rather than o CD4+ T helper cells).

b) Infectious agents and compartmentalised immune responses

It is hypothesised that an infectious organism located in the adjacent meninges or an infectious agent with primary tropism for cortical neurons and/or oligodendrocytes may result in formation of aberrant tertiary lymphoid-like structures within the meninges or the cortical layer and may induce a chronic compartmentalised inflammatory response against 
neurons or oligodendrocytes that is characterised by the presence of CD4+ and CD8+ Tcells and CD20+ B-cells and plasmablasts.

\section{CNS innate inflammatory mechanisms}

a) Chronic Microglial Activation

The presence of a chronic inflammation in the subarachnoid and perivascular spaces, environmental factors such as toxins, or neuronal damage or death, might cause an abnormal and chronic microglial over-activation that can be directly harmful to neurons and may also lead to a dysregulation of astrocyte functions and glutamate metabolism resulting in further neuronal and synaptic damage.

\section{Non-inflammatory neurodegenerative mechanisms}

a) Primary neurodegeneration

A primary neurodegenerative event involving neurons, astrocytes or oligodendrocytes that may be caused or worsened by mitochondrial injury and metabolic compromise may explain the substantial neuronal injury with relatively minor parenchymal inflammation, microglial activation and associated meningeal inflammation, which are usually observed in multiple sclerosis.

a) Retrograde degeneration due to white matter damage

Reduction of cortical grey matter volume and thinning may be the consequence of white matter pathology via retrograde degeneration especially in the more advanced disease phase, thus explaining the significant neuronal loss observed even in the non-demyelinated cortex.

\section{BOX 2: Role of mitochondrial injury in grey matter damage}

Mitochondrial injury can be found in lesions characterized by all of the typical pathological features of multiple sclerosis including inflammation, demyelination, oligodendrocyte apoptosis, and axon degeneration ${ }^{119}$. A role for inflammation in mitochondrial dysfunction is suggested by post-mortem studies showing that reactive oxygen species (ROS) produced by activated microglia and macrophages can induce mitochondrial dysfunction not only in the white matter ${ }^{120}$, but also in the grey matter lesions ${ }^{104}$. Several lines of evidence have led to the hypothesis that mitochondrial injury is a primary phenomenon in multiple sclerosis ${ }^{121}$.

a. Respiratory-chain deficient neurons have been identified in normal-appearing brain tissue $^{122}$.

b. Respiratory-deficient neurons with multiple deletions in mitochondrial DNA are distributed diffusely in the cortex of MS patients ${ }^{122}$. 
c. Focal intra-axonal mitochondrial pathology is the earliest sign of damage in a mouse model of $\mathrm{MS}^{123}$.

d. Abnormal mitochondrial gene expression and impaired activities of mitochondrial respiratory chain complexes I and III, which can be found in demyelinated cortex samples of progressive MS patients ${ }^{124}$, may result in mitochondrial dysfunction and a state of impaired energy production.

e. A characteristic hypoxia-like injury caused by mitochondrial impairment might be involved in neurodegeneration in MS lesions ${ }^{125126}$.

Irrespective of the initial event, the presence of a local energy deficit in grey matter may induce a vicious cycle that leads to increased mitochondrial production ROS and further deletions of mitochondrial $\mathrm{DNA}^{127}$. Energy-deficient neurons may be more prone to inflammatory insult, which induces increased energy demand in the presence of a reduced energy supply ${ }^{128}$. Although the precise causes of neuronal respiratory deficiency in MS are yet unclear, the neuronal energy deficit is crucial for inducing axonal swelling and subsequent neuronal death, especially when it occurs as a consequence of inflammation, as is the case in grey matter regions in multiple sclerosis patients.

\section{Acknowledgements:}

M. Calabrese is supported by the Progressive MS Alliance (PA-0124)

R Magliozzi is supported by the Italian MS Foundation grant (FISM 2011/R/23) and by the Italian Ministry of Health grant (GR-2010-2313255).

R. Reynolds is supported by the UK Multiple Sclerosis Society and Medical Research Council.

R. Martin and the Neuroimmunology and MS Research Section are supported by the Clinical Research Priority Program MS (CRPP $\left.{ }^{\mathrm{MS}}\right)$ of the University Zurich, the Swiss National Science Foundation (SNF), a European Research Council (ERC) Advanced Grant, the EU-FP7 framework program and the Swiss MS Society.

\section{Referencees}

1. Noseworthy JH, Lucchinetti C, Rodriguez M, Weinshenker BG. Multiple sclerosis. N Engl J Med 2000 Sep 28;343(13):938-52. Review. 
2. Sospedra M and Martin R. Immunology of Multiple Sclerosis. Annu. Rev. Immunol 2005 $23 ; 683-747$.

3. Lill CM et al. CXCR5, SOX8, RPS6KB1 and ZBTB46 are genetic risk loci for multiple sclerosis. Brain. 2013 Jun;136(Pt 6):1778-82.

4. Ascherio A. Environmental factors in multiple sclerosis. Expert Rev Neurother. 2013 Dec;13(12 Suppl):3-9.

5. Dutta R, Trapp BD. [Pathology and definition of multiple sclerosis]. Rev Prat. 2006 Jun 30;56(12):1293-8. Review.

6. Lassmann H, Brück W, Lucchinetti CF. The immunopathology of multiple sclerosis: an overview. Brain Pathol. 2007 Apr;17(2):210-8. Review.

7. Dawson JD. The Histology of Disseminated Sclerosis. Trans Royal Soc Edin. 1916; 50: 517740.

8. Brownell B, Hughes JT. The distribution of plaques in the cerebrum in multiple sclerosis. $\mathrm{J}$ Neurol Neurosurg Psychiatry. 1962; 25: 315-20.

9. Chard D, Miller D. Grey matter pathology in clinically early multiple sclerosis: evidence from magnetic resonance imaging. J Neurol Sci. 2009 Jul 15;282(1-2):5-11.

10. Calabrese M, Filippi M, Gallo P. Cortical lesions in multiple sclerosis. Nat Rev Neurol. 2010 Aug;6(8):438-44.

11. Kidd D, Barkhof F, McConnell R, Algra PR, Allen IV, Revesz T. Cortical lesions in multiple sclerosis. Brain 1999; 122: 17-26.

12. Peterson JW, Bö L, Mörk S, Chang A, Trapp BD. Transected neuritis, apoptotic neurons and reduced inflammation in cortical multiple sclerosis lesions. Ann Neurol 2001; 50: 389-400

* The seminal paper on cortical lesions in multiple sclerosis

13. Bö L, Vedeler CA, Nyland HI, Trapp BD, Mörk SJ. Subpial demyelination in the cerebral cortex of multiple sclerosis patients. J Neuropathol Exp Neurol 2003; 62: 723-32.

14. Brink BP, Veerhuis R, Breij EC, van der Valk P, Dijkstra CD, Bö L. The pathology of multiple sclerosis is location-dependent: no significant complement activation is detected in purely cortical lesions. J Neuropathol Exp Neurol. 2005 Feb;64(2):147-55.

15. van Horssen J, Brink BP, de Vries HE, van der Valk $\mathrm{P}, \mathrm{B} \varnothing \mathrm{L}$. The blood-brain barrier in cortical multiple sclerosis lesions. J Neuropathol Exp Neurol. 2007 Apr;66(4):321-8.

16. Wegner C, Esiri MM, Chance SA, Palace J, Matthews PM. Neocortical neuronal, synaptic, and glial loss in multiple sclerosis. Neurology. 2006 Sep 26;67(6):960-7.

17. Magliozzi R., et al. A gradient of neuronal loss and meningeal inflammation in multiple sclerosis. Ann Neurol. 2010 Oct;68(4):477.

* A very interesting study showing a direct relationship between meningeal inflammation, subpial demyelination and neuronal loss in MS 
18. Freund $\mathrm{P}$, et al. Disability, atrophy and cortical reorganization following spinal cord injury. Brain 134:1610-22 (2011).

19. Sailer M, et al Focal thinning of the cerebral cortex in multiple sclerosis. Brain. 2003 Aug;126(Pt 8):1734-44.

20. Narayanan S, et al. Imaging of axonal damage in multiple sclerosis: spatial distribution of magnetic resonance imaging lesions. Ann Neurol. 1997 Mar;41(3):385-91.

21. Varga AW, Johnson G, Babb JS, Herbert J, Grossman RI, Inglese M. White matter hemodynamic abnormalities precede sub-cortical gray matter changes in multiple sclerosis. $\mathrm{J}$ Neurol Sci. 2009 Jul 15;282(1-2):28-33.

22. Dziedzic T, et al. Wallerian degeneration: a major component of early axonal pathology in multiple sclerosis. Brain Pathol. 2010 Sep;20(5):976-85. do

23. Henry RG, Shieh M, Amirbekian B, Chung S, Okuda DT, Pelletier D. Connecting white matter injury and thalamic atrophy in clinically isolated syndromes. J Neurol Sci. 282:61-6 (2009).

24. Audoin B, Davies GR, Finisku L, Chard DT, Thompson AJ, Miller DH. Localization of grey matter atrophy in early RRMS: A longitudinal study. J Neurol. 253:1495-501 (2006).

25. De Stefano N, at al. Evidence of early cortical atrophy in MS: relevance to white matter changes and disability. Neurology 60:1157-62 (2003).

* One of the seminal MRI studies on cortical atrophy in multiple sclerosis

26. Furby J, et al. Different white matter lesion characteristics correlate with distinct grey matter abnormalities on magnetic resonance imaging in secondary progressive multiple sclerosis. Mult Scler. 15:687-94 (2009)

27. Sanfilipo MP, Benedict RH, Sharma J, Weinstock-Guttman B, Bakshi R. The relationship between whole brain volume and disability in multiple sclerosis: a comparison of normalized gray vs. white matter with misclassification correction. Neuroimage 26:1068-77 (2005).

28. Roosendaal SD, et al. Grey matter volume in a large cohort of MS patients: relation to MRI parameters and disability. Mult Scler. 17:1098-106 (2011).

29. Ceccarelli A, et al. A voxel-based morphometry study of grey matter loss in MS patients with different clinical phenotypes. Neuroimage. 42:315-22 (2008).

30. Battaglini $\mathrm{M}$, et al. Voxel-wise assessment of progression of regional brain atrophy in relapsing-remitting multiple sclerosis. J Neurol Sci. 282:55-60 (2009).

31. Bendfeldt $\mathrm{K}$, et al. Association of regional gray matter volume loss and progression of white matter lesions in multiple sclerosis - A longitudinal voxel-based morphometry study. Neuroimage. 45:60-7 (2009).

32. Pagani E, et al. Regional brain atrophy evolves differently in patients with multiple sclerosis according to clinical phenotype. AJNR Am J Neuroradiol. 26:341-6 (2005). 
33. Sepulcre J, et al. Contribution of white matter lesions to gray matter atrophy in multiple sclerosis: evidence from voxel-based analysis of T1 lesions in the visual pathway. Arch Neurol. 66:173-9 (2009).

34. Gilmore CP, Donaldson I, Bö L, Owens T, Lowe J, Evangelou N. Regional variations in the extent and pattern of grey matter demyelination in multiple sclerosis: a comparison between the cerebral cortex, cerebellar cortex, deep grey matter nuclei and the spinal cord. J Neurol Neurosurg Psychiatry. 2009 Feb;80(2):182-7.

35. Kutzelnigg A, et al. Widespread demyelination in the cerebellar cortex in multiple sclerosis. Brain Pathol. 2007 Jan;17(1):38-44.

36. Gilmore CP, et al. Spinal cord neuronal pathology in multiple sclerosis. Brain Pathol. 2009 Oct;19(4):642-9.

37. Howell, O. et al. Meningeal inflammation is widespread and linked to cortical pathology in multiple sclerosis. Brain. 2011 Sep;134(Pt 9):2755.

38. Lucchinetti CF, et al. Inflammatory cortical demyelination in early multiple sclerosis. N Engl J Med. 2011 Dec 8;365(23):2188

* The first neuropathological characterization of inflammatory cortical lesions in early multiple sclerosis.

39. Calabrese $\mathrm{M}$, et al. Detection of cortical inflammatory lesions by double inversion recovery magnetic resonance imaging in patients with multiple sclerosis. Arch Neurol. 64:1416-22 (2007).

40. Giorgio A, et al. Cortical lesions in radiologically isolated syndrome. Neurology. 2011 Nov 22;77(21):1896-9.

41. Calabrese M, Gallo P. Magnetic resonance evidence of cortical onset of multiple sclerosis. Mult Scler. 15:933-41 (2009).

42. Seewann A, et al. Imaging the tip of the iceberg: visualization of cortical lesions in multiple sclerosis. Mult Scler. 2011 Oct;17(10):1202-10.

43. Chard DT, Griffin CM, Parker GJ, Kapoor R, Thompson AJ, Miller DH. Brain atrophy in clinically early relapsing-remitting multiple sclerosis. Brain 125:327-37 (2002).

44. Tiberio M, et al. Gray and white matter volume changes in early RRMS: a 2-year longitudinal study. Neurology 64:1001-7 (2005).

45. Sbardella E, et al. Assessing the correlation between grey and white matter damage with motor and cognitive impairment in multiple sclerosis patients. PLoS One. 2013 May 16;8(5):e63250.

46. Steenwijk MD, et al What Explains Gray Matter Atrophy in Long-standing Multiple Sclerosis? Radiology. 2014 Apr 23:132708.

47. Calabrese $\mathrm{M}$, et al. Imaging distribution and frequency of CLs in patients with multiple sclerosis. Neurology 75:1234-40 (2010).

48. Vercellino M, et al. Demyelination, inflammation, and neurodegeneration in multiple sclerosis deep gray matter. J Neuropathol Exp Neurol. 68:489-502 (2009). 
49. Geurts JJ, et al. Extensive hippocampal demyelination in multiple sclerosis. J Neuropathol Exp Neurol. 66:819-27 (2007).

50. Cohen-Adad J, et al. In vivo evidence of disseminated subpial T2* signal changes in multiple sclerosis at 7 T: a surface-based analysis. Neuroimage. 57:55-62 (2011)

51. Audoin B, et al. Atrophy mainly affects the limbic system and the deep grey matter at the first stage of multiple sclerosis. J Neurol Neurosurg Psychiatry 81:690-5 (2010).

52. Bendfeldt K, et al. Spatiotemporal distribution pattern of white matter lesion volumes and their association with regional grey matter volume reductions in relapsing-remitting multiple sclerosis. Hum Brain Mapp. 31:1542-55 (2010).

53. Bettelli E, Pagany M, Weiner HL, Linington C, Sobel RA, Kuchroo VK. Myelin oligodendrocyte glycoprotein-specific $\mathrm{T}$ cell receptor transgenic mice develop spontaneous autoimmune optic neuritis. J Exp Med. 2003 May 5;197(9):1073-81.

54. Krishnamoorthy $\mathrm{G}$, et al. Myelin-specific $\mathrm{T}$ cells also recognize neuronal autoantigen in a transgenic mouse model of multiple sclerosis. Nat Med. 2009 Jun;15(6):626-32

55. Serafini B, Rosicarelli B, Magliozzi R, Stigliano E, Aloisi F. Detection of ectopic B-cell follicles with germinal centers in the meninges of patients with secondary progresive multiple sclerosis. Brain Pathol. 2004; 14: 164-74;

56. Ascherio A, Munger KL. Epstein-barr virus infection and multiple sclerosis: a review. J Neuroimmune Pharmacol. 2010 Sep;5(3):271-7.

57. Ascherio A1, et al. Epstein-Barr virus antibodies and risk of multiple sclerosis: a prospective study.JAMA. 2001 Dec 26;286(24):3083-8.

58. Levin LI1, et al. Multiple sclerosis and Epstein-Barr virus. JAMA. 2003 Mar 26;289(12):15336.

59. Serafini B, Rosicarelli B, Franciotta D, Magliozzi R, Reynolds R, Cinque P, Andreoni L, Trivedi P, Salvetti M, Faggioni A, Aloisi F. Dysregulated Epstein-Barr virus infection in the multiple sclerosis brain. J Exp Med. 2007; 204(12):2899-2912.

60. Serafini B, Muzio L, Rosicarelli B, Aloisi F. Radioactive in situ hybridization for Epstein-Barr virus-encoded small RNA supports presence of Epstein-Barr virus in the multiple sclerosis brain. Brain. 2013 Jul;136(Pt 7):e233.

61. Magliozzi R, et al. B-cell enrichment and Epstein-Barr virus infection in inflammatory cortical lesions in secondary progressive multiple sclerosis. J Neuropathol Exp Neurol. 2013 Jan;72(1):29-41.

62. Angelini DF, et al. Increased CD8+ T cell response to Epstein-Barr virus lytic antigens in the active phase of multiple sclerosis. PLoS Pathog. 2013;9(4).

63. Lossius A, et al High-throughput sequencing of TCR repertoires in multiple sclerosis reveals intrathecal enrichment of EBV-reactive CD8(+) T cells. Eur J Immunol. 2014 Nov;44(11):3439-52. 
64. Sargsyan SA, et al. Absence of Epstein-Barr virus in the brain and CSF of patients with multiple sclerosis. Neurology. 2010 Apr 6;74(14):1127-35

65. Willis $\mathrm{SN}$, et al. Epstein-Barr virus infection is not a characteristic feature of multiple sclerosis brain. Brain. 2009 Dec;132(Pt 12):3318-28.

66. Lassmann H(1), Niedobitek G, Aloisi F, Middeldorp JM; NeuroproMiSe EBV Working Group. Epstein-Barr virus in the multiple sclerosis brain: a controversial issue-report on a focused workshop held in the Centre for Brain Research of the Medical University of Vienna, Austria. Brain. 2011 Sep;134(Pt 9):2772-86.

67. Aloisi F, Serafini B, Magliozzi R, Howell OW, Reynolds R. Detection of Epstein-Barr virus and B-cell follicles in the multiple sclerosis brain: what you find depends on how and where you look. Brain 2010;133:e157.

68. Maggi F1, et al. Low prevalence of TT virus in the cerebrospinal fluid of viremic patients with central nervous system disorders. J Med Virol. 2001 Oct;65(2):418-22.

69. Sospedra M, et al. Recognition of conserved amino acid motifs of common viruses and its role in autoimmunity. PLoS Pathog. 2005 Dec;1(4):e41

70. Lamberto I, Gunst K, Müller H, Zur Hausen H, de Villiers EM. Mycovirus-like DNA virus sequences from cattle serum and human brain and serum samples from multiple sclerosis patients. Genome Announc. 2014 Aug 28;2(4).

71. Borkosky SS, Whitley C, Kopp-Schneider A, zur Hausen H, de Villiers EM. Epstein-Barr virus stimulates torque teno virus replication: a possible relationship to multiple sclerosis. PLoS One. 2012;7(2):e32160.

72. Zeis T, Graumann U, Reynolds R, Schaeren-Wiemers N. Normal-appearing white matter in multiple sclerosis is in a subtle balance between inflammation and neuroprotection. Brain. 2008 Jan;131(Pt 1):288-303.

73. Baranzini SE, et al. Genetic variation influences glutamate concentrations in brains of patients with multiple sclerosis. Brain. 2010 Sep;133(9):2603-11

74. Mastronardi FG, Moscarello MA. Molecules affecting myelin stability: a novel hypothesis regarding the pathogenesis of multiple sclerosis. J Neurosci Res. 2005 May 1;80(3):301-8.

75. Neumann H, Cavalié A, Jenne DE, Wekerle H. Induction of MHC class I genes in neurons. Science. 1995 Jul 28;269(5223):549-52.

76. Jersild C, Fog T, Hansen GS, Thomsen M, Svejgaard A, Dupont B. Histocompatibility determinants in multiple sclerosis, with special reference to clinical course. Lancet. 1973 Dec 1;2(7840):1221-5.

77. IMSGC, Sawcer S, et al. Genetic risk and a primary role for cell-mediated immune mechanisms in multiple sclerosis. Nature. 2011 Aug 10;476(7359):214-9.

78. Martin R, et al. Fine specificity and HLA restriction of myelin basic protein-specific cytotoxic $\mathrm{T}$ cell lines from multiple sclerosis patients and healthy individuals. J Immunol. $1990 \mathrm{Jul}$ $15 ; 145(2): 540-8$. 
79. Ota K, Matsui M, Milford EL, Mackin GA, Weiner HL, Hafler DA. T-cell recognition of an immunodominant myelin basic protein epitope in multiple sclerosis. Nature. $1990 \mathrm{Jul}$ $12 ; 346(6280): 183-7$

80. Bielekova B, Sung MH, Kadom N, Simon R, McFarland H, Martin R. Expansion and functional relevance of high-avidity myelin-specific CD4+ $\mathrm{T}$ cells in multiple sclerosis. $\mathrm{J}$ Immunol. 2004 Mar 15;172(6):3893-904

81. Yates RL, Esiri MM, Palace J, Mittal A, DeLuca GC. The influence of HLA-DRB1*15 on motor cortical pathology in multiple sclerosis. Neuropathol Appl Neurobiol. 2014 Jun 25.

82. Höftberger R, et al.. Expression of major histocompatibility complex class I molecules on the different cell types in multiple sclerosis lesions. Brain Pathol. 2004 Jan;14(1):43-50.

83. Babbe $\mathrm{H}$, et al. Clonal expansions of $\mathrm{CD} 8(+) \mathrm{T}$ cells dominate the $\mathrm{T}$ cell infiltrate in active multiple sclerosis lesions as shown by micromanipulation and single cell polymerase chain reaction. J Exp Med. 2000 Aug 7;192(3):393-404.

84. Ulvestad E, Williams K, Bø L, Trapp B, Antel J, Mørk S. HLA class II molecules (HLA-DR, DP, -DQ) on cells in the human CNS studied in situ and in vitro. Immunology. 1994 Aug;82(4):535-41.

85. Liblau RS, Gonzalez-Dunia D, Wiendl H, Zipp F. Neurons as targets for T cells in the nervous system. Trends Neurosci. 2013 Jun;36(6):315-24.

86. Meuth SG, et al. Cytotoxic CD8+ T cell-neuron interactions: perforin-dependent electrical silencing precedes but is not causally linked to neuronal cell death. J Neurosci. 2009 Dec 9;29(49):15397-409.

87. Suidan HS, Bouvier J, Schaerer E, Stone SR, Monard D, Tschopp J. Granzyme A released upon stimulation of cytotoxic $\mathrm{T}$ lymphocytes activates the thrombin receptor on neuronal cells and astrocytes. Proc Natl Acad Sci U S A. 1994 Aug 16;91(17):8112-6.

88. Medana IM, Gallimore A, Oxenius A, Martinic MM, Wekerle H, Neumann H. MHC class Irestricted killing of neurons by virus-specific CD8+ T lymphocytes is effected through the Fas/FasL, but not the perforin pathway,. Eur J Immunol. 2000 Dec;30(12):3623-33.

89. Aktas $\mathrm{O}$, et al. Neuronal damage in autoimmune neuroinflammation mediated by the death ligand TRAIL. Neuron. 2005 May 5;46(3):421-32.

90. Mizuno T, et al. Interferon-gamma directly induces neurotoxicity through a neuron specific, calcium-permeable complex of IFN-gamma receptor and AMPA GluR1 receptor. FASEB J. 2008 Jun;22(6):1797-806.

91. Vergelli M, et al. Human autoreactive CD4+ T cell clones use perforin- or Fas/Fas ligandmediated pathways for target cell lysis. J Immunol. 1997 Mar 15;158(6):2756-61.

92. Vergelli M, Le H, van Noort JM, Dhib-Jalbut S, McFarland H, Martin R. A novel population of CD4+CD56+ myelin-reactive $\mathrm{T}$ cells lyses target cells expressing CD56/neural cell adhesion molecule. J Immunol. 1996 Jul 15;157(2):679-88.

93. Zaguia F, et al. Cytotoxic NKG2C+CD4 T cells target oligodendrocytes in multiple sclerosis. J Immunol. 2013 Mar 15;190(6):2510-8. 
94. Magliozzi R, et al. Meningeal B-cell follicles in secondary progressive multiple sclerosis associate with early onset of disease and severe cortical pathology. Brain. 2007 Apr;130(Pt 4):1089-104.

95. Magliozzi R, Columba-Cabezas S, Serafini B, Aloisi F. Intracerebral expression of CXCL13 and BAFF is accompanied by formation of lymphoid follicle-like structures in the meninges of mice with relapsing experimental autoimmune encephalomyelitis. J Neuroimmunol. 2004 Mar;148(1-2):11-23;

96. Peters A, et al. Th17 cells induce ectopic lymphoid follicles in central nervous system tissue inflammation. Immunity. 2011 Dec 23;35(6):986-96.

97. Choi SR, et al. Meningeal inflammation plays a role in the pathology of primary progressive multiple sclerosis. Brain. 2012 Oct;135(Pt 10):2925-37.

98. Kramann N, et al. Increased Meningeal T and Plasma Cell Infiltration Is Associated with Early Subpial Cortical Demyelination in Common Marmosets with Experimental Autoimmune Encephalomyelitis. Brain Pathol. 2014 Jul 18

99. Gardner C, Magliozzi R, Durrenberger PF, Howell OW, Rundle J, Reynolds R. Cortical grey matter demyelination can be induced by elevated pro-inflammatory cytokines in the subarachnoid space of MOG-immunized rats. Brain. 2013 Oct 30

100.Kooi EJ, Geurts JJ, van Horssen J, Bø L, van der Valk P. Meningeal inflammation is not associated with cortical demyelination in chronic multiple sclerosis. J Neuropathol Exp Neurol. 2009 Sep;68(9):1021-8.

101.Guseo A, Jellinger K. The significance of perivascular infiltrations in multiple sclerosis. J Neurol. 1975 Dec 2;211(1):51-60.

102.Kutzelnigg A, et al. Cortical demyelination and diffuse white matter injury in multiple sclerosis. Brain. 2005 Nov;128(Pt 11):2705-12.

* Elegant autoptic evidence of white matter and grey matter damage in multiple sclerosis.

103.Reynolds R, Roncaroli F, Nicholas R, Radotra B, Gveric D, Howell O. The neuropathological basis of clinical progression in multiple sclerosis. Acta Neuropathol. 2011 Aug;122(2):155-70.

104.Fischer MT, et al. Disease-specific molecular events in cortical multiple sclerosis lesions. Brain. 2013 Jun;136(Pt 6):1799-815.

105.Calabrese $\mathrm{M}$, et al. The changing clinical course of multiple sclerosis: a matter of gray matter. Ann Neurol. 2013 Jul;74(1):76-83.

* Interesting paper showing that grey matter damage is implicated in the progression of clinical disability during the course of multiple sclerosis

106.Androdias G, Reynolds R, Chanal M, Ritleng C, Confavreux C, Nataf S. Meningeal T cells associate with diffuse axonal loss in multiple sclerosis spinal cords. Ann Neurol. 2010;68(4):465.

107.Dutta R, Trapp BD. Pathogenesis of axonal and neuronal damage in multiple sclerosis. Neurology. 2007 May 29;68(22 Suppl 3):S22-31; discussion S43-54. 
108.Kreutzberg GW. Microglia: a sensor for pathological events in the CNS. Trends Neurosci. 1996 Aug;19(8):312-8. Review. Nimmerjahn et al 2005

109.Nimmerjahn A, Kirchhoff F, Helmchen F. Resting microglial cells are highly dynamic surveillants of brain parenchyma in vivo. Science. 2005 May 27;308(5726):1314-8.

110.Aloisi F. Immune function of microglia. Glia. 2001 Nov;36(2):165-79. Review.

111.Block ML, Hong JS. Chronic microglial activation and progressive dopaminergic neurotoxicity. Biochem Soc Trans. 2007 Nov;35(Pt 5):1127-32. Review.;

112.Polazzi E, Contestabile A. Reciprocal interactions between microglia and neurons: from survival to neuropathology. Rev Neurosci. 2002;13(3):221-42.

113.Gray E, Thomas TL, Betmouni S, Scolding N, Love S. Elevated matrix metalloproteinase-9 and degradation of perineuronal nets in cerebrocortical multiple sclerosis plaques. $\mathrm{J}$ Neuropathol Exp Neurol. 2008 Sep;67(9):888-99.

114. Vercellino $\mathrm{M}$, et al. Altered glutamate reuptake in relapsing-remitting and secondary progressive multiple sclerosis cortex: correlation with microglia infiltration, demyelination, and neuronal and synaptic damage. J Neuropathol Exp Neurol. 2007 Aug;66(8):732-9

115.Kooi EJ, Strijbis EM, van der Valk P, Geurts JJ. Heterogeneity of cortical lesions in multiple sclerosis: clinical and pathologic implications. Neurology. 2012 Sep 25;79(13):1369-76.

116.Stys PK, Zamponi GW, van Minnen J, Geurts JJ. Will the real multiple sclerosis please stand up? Nat Rev Neurosci. 2012 Jun 20;13(7):507-14.

117.Barnett MH, Prineas JW. Relapsing and remitting multiple sclerosis: pathology of the newly forming lesion. Ann Neurol. 2004 Apr;55(4):458-68.

118.Henderson AP, Barnett MH, Parratt JD, Prineas JW. Multiple sclerosis: distribution of inflammatory cells in newly forming lesions. Ann Neurol. 2009 Dec;66(6):739-53

119.Lassmann H, van Horssen J, Mahad D. Progressive multiple sclerosis: pathology and pathogenesis. Nat Rev Neurol. 2012 Nov 5;8(11):647-56.

* A comprehensive review on pathology and pathogenesis of progressive multiple sclerosis

120.Fischer MT, et al. NADPH oxidase expression in active multiple sclerosis lesions in relation to oxidative tissue damage and mitochondrial injury. Brain. 2012 Mar;135(Pt 3):886-99.

121.Witte ME, Geurts JJ, de Vries HE, van der Valk P, van Horssen J. Mitochondrial dysfunction: a potential link between neuroinflammation and neurodegeneration? Mitochondrion. 2010 Aug;10(5):411-8.

122. Campbell GR, et al. Mitochondrial DNA deletions and neurodegeneration in multiple sclerosis. Ann Neurol. 2011 Mar;69(3):481-92.

123. Nikić I et al. A reversible form of axon damage in experimental autoimmune encephalomyelitis and multiple sclerosis. Nat Med. 2011 Apr;17(4):495-9.

124.van Horssen J, Witte ME, Ciccarelli O. The role of mitochondria in axonal degeneration and tissue repair in MS. Mult Scler. 2012 Aug;18(8):1058-67., 
125.Mahad D, Ziabreva I, Lassmann H, Turnbull D. Mitochondrial defects in acute multiple sclerosis lesions. Brain. 2008 Jul;131(Pt 7):1722-35..

126. Marik C, Felts PA, Bauer J, Lassmann H, Smith KJ. Lesion genesis in a subset of patients with multiple sclerosis: a role for innate immunity? Brain. 2007 Nov;130(Pt 11):2800-15.

127.Druzhyna NM, Wilson GL, LeDoux SP. Mitochondrial DNA repair in aging and disease. Mech Ageing Dev 2008;129:383-390

128. Trapp BD. Virtual hypoxia and chronic necrosis of demyelinated axons in multiple sclerosis. Lancet Neurol. 2009 Mar;8(3):280-91.

\section{Suggested Glossary Terms}

Magnetic resonance imaging (MRI): A non-invasive method used to obtain images of living tissue. It uses radio-frequency pulses and magnetic field gradients; the principle of nuclear magnetic resonance is used to reconstruct images of tissue characteristics (for example, proton density or water diffusion parameters).

Complement: A set of plasma proteins that attack extracellular pathogens. The pathogen becomes coated with complement proteins that facilitate pathogen removal by phagocytes

Wallerian degeneration: The degeneration of an axon distal to a site of injury, which begins to occur about 1.5 days after a lesion.

Clinically isolated syndrome (CIS): patient having just the first clinical episode suggestive of a inflammatory demyelinating disease of the central nervous system

Relapsing remitting multiple sclerosis (RRMS): early phase of Multiple Sclerosis characterized by several neurological episodes followed by complete or incomplete recovery

Radiologically isolated syndrome: patients having the radiological evidence of an inflammatory demyelinating disease of the central nervous system but no signs or symptoms of such disease.

Primary and secondary progressive disease: phases of multiple sclerosis characterized by a slow progression of disability without a well defined clinical relapse. They usually follow the relapsing remitting phase (secondary progressive phase) but they can also be the first phase of the disease (primary progressive multiple sclerosis)

T cells: a lymphocyte type that is responsible for cell-mediated immune responses by providing help (e.g. cytokines) to other immune cells or by cytotoxicity, e.g. killing of a virus-infected cell.

B cells: a lymphocyte type that expresses immunoglobulins as surface receptor or, when they are fully mature after antigenic stimulation, release antibodies, which are e.g. directed against a virus or bacteria.

Natural killer cells: a white blood cell population that does not express antigen-specific recognition receptors like T- and B cells, but recognizes e.g. virus-infected cells that express less or no HLA-class I molecules. Natural killer (NK) cells are important in controlling viral infections and recognition of mutated (tumor) cells.

Experimental autoimmune encephalitis (EAE): An animal model of multiple sclerosis that is initiated in animals by injecting myelin proteins or peptides to raise autoreactive $\mathrm{T}$ cells, or by the transfer of autoreactive $\mathrm{T}$ cells into naive recipients. 

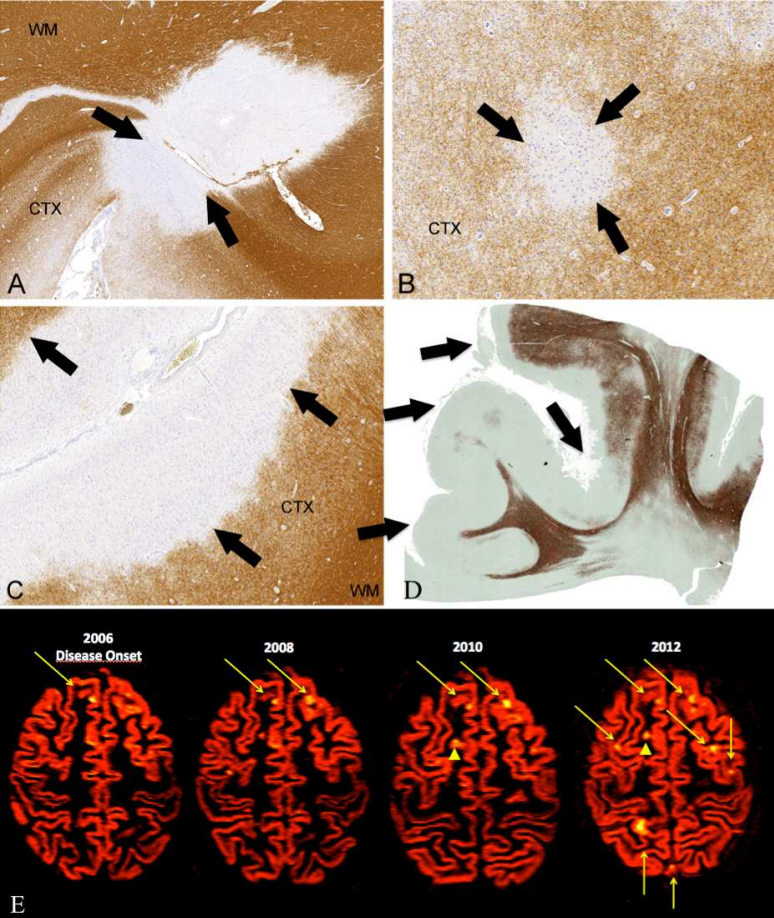
Inflammatory neuronal damage
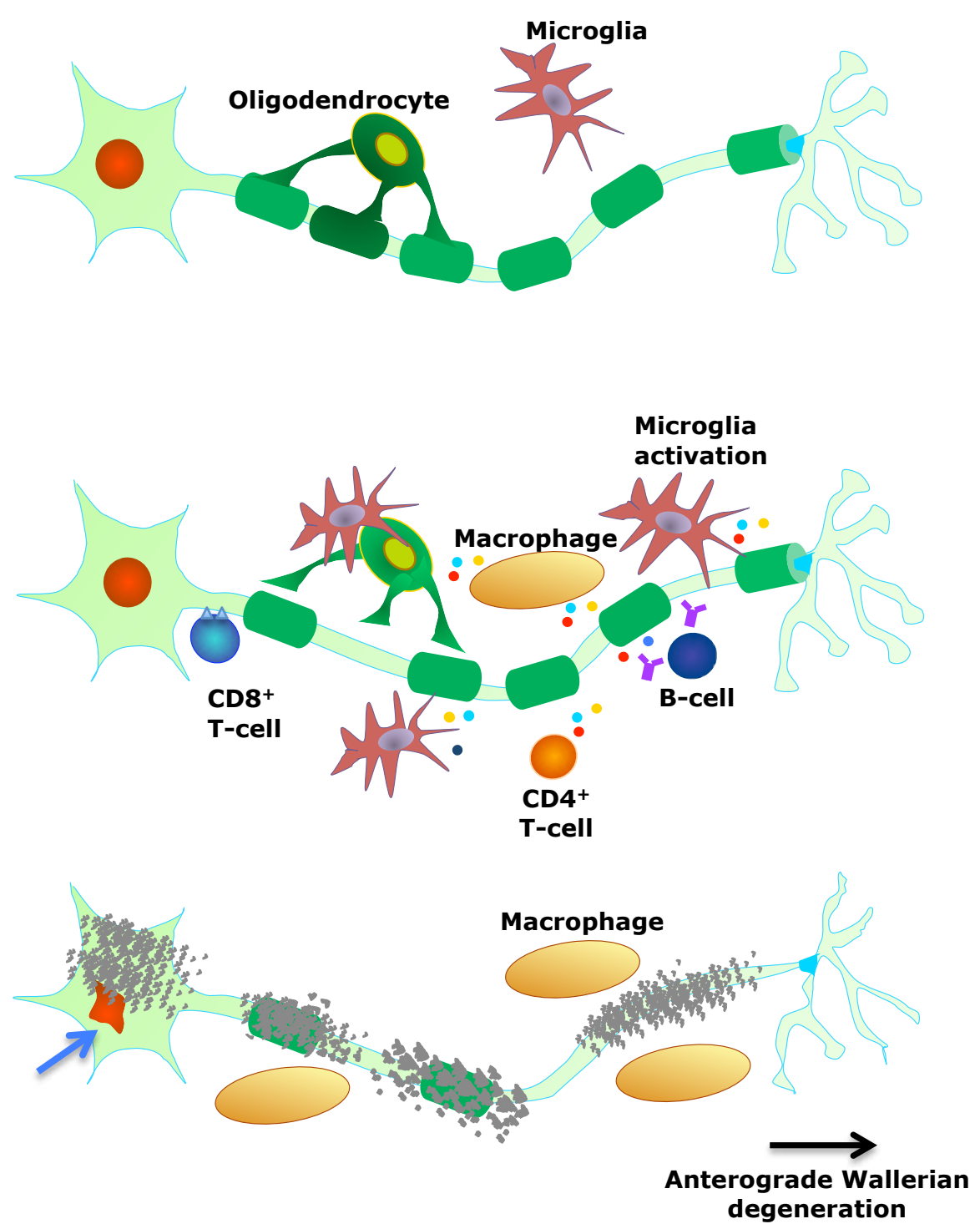

Non-inflammatory neuronal damage
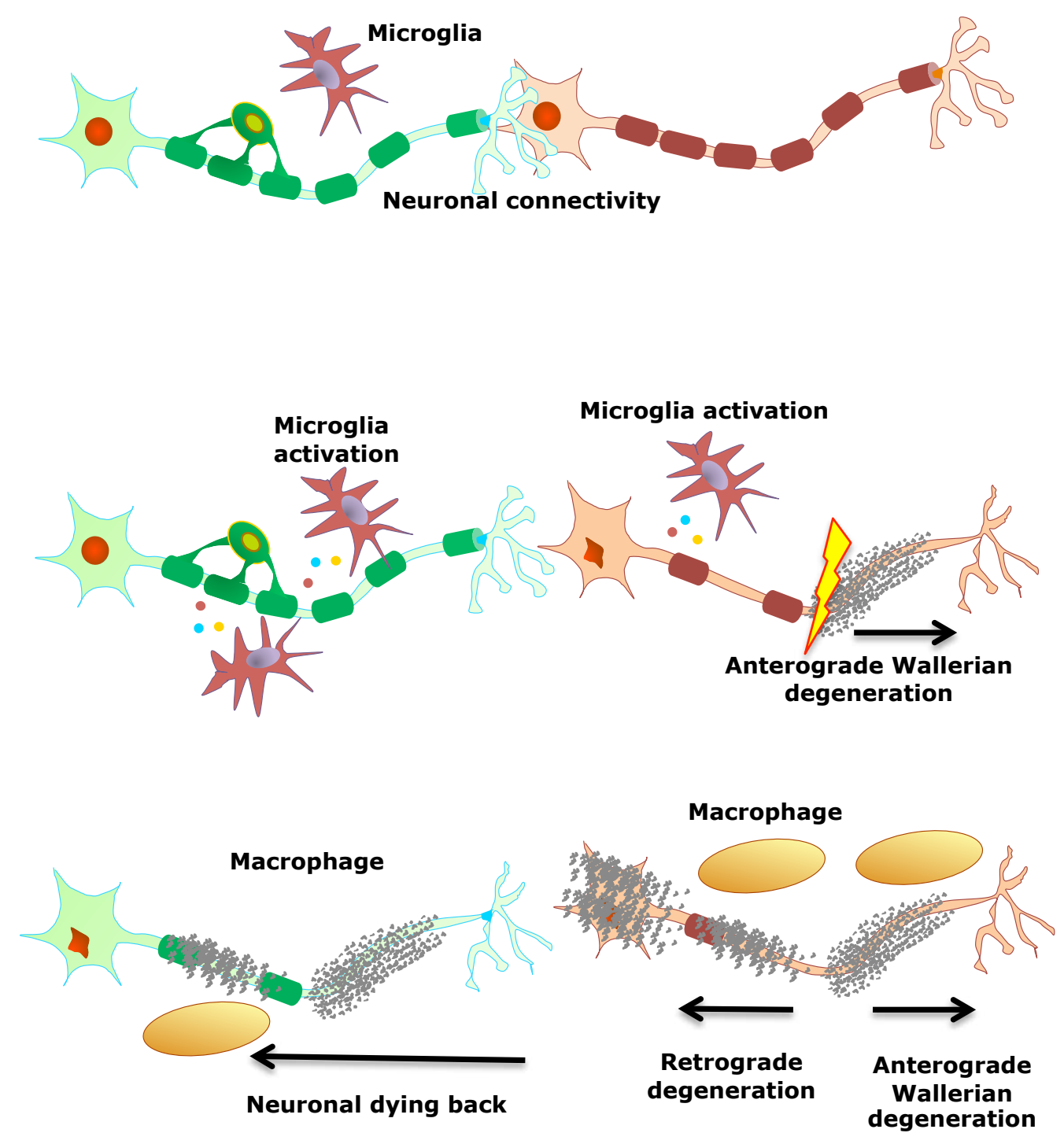


\section{Figure 2}

Inflammatory neurodegeneration. Release of pro-inflammatory and cytotoxic molecules, as well as cell contact dependent mechanisms of T-cell mediated damage, may directly or indirectly, by mediating microglia/macrophage activation and oligodendrocyte injury, give rise to death of neuronal cell body and nuclei ( blue arrow indicating morphology alteration of the characteristic picnotic nuclei), dendrites shrinkage and axonal degeneration in the cerebral cortex. This could in turn lead to dysfunction of the downstream neuronal network (anterograde transynaptic degeneration).

Non-inflammatory neurodegeneration. Retrograde degeneration could impair neuronal functions through retrograde damage propagating backwards and leading to cell death of cortical neurons whose axons have been impaired in white matter lesions and along the corticospinal tract. Injury at the terminal endings could spreads back towards the cell body and lead to neuronal dying-back. Finally, even in these cases activation of microglia/ macrophage indeed contribute to the exacerbation of the damage. 



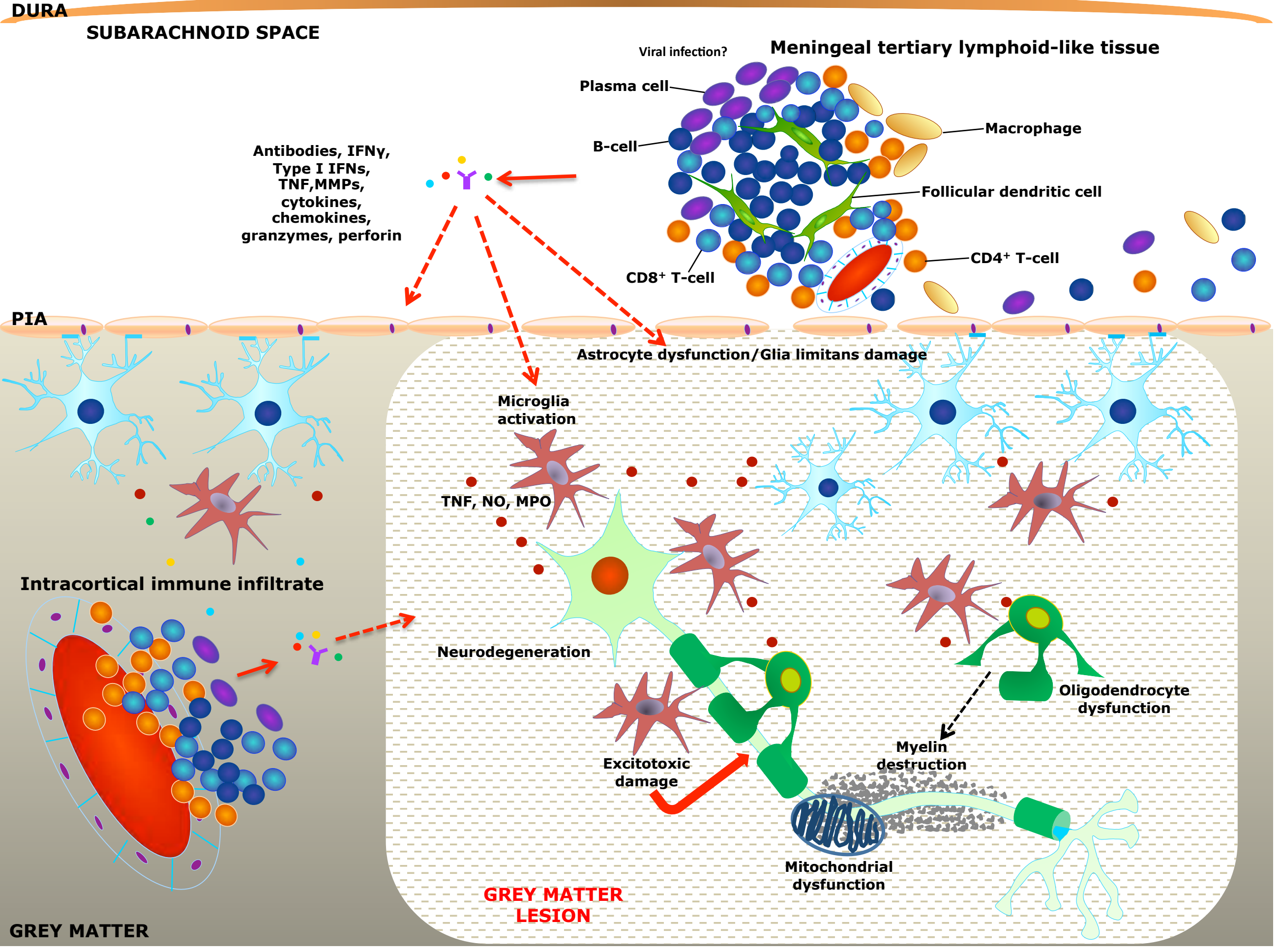

